\title{
AI-BCDCP: ARTIFICIALLY INTELLIGENT BASE STATION CONTROLLED DYNAMIC CLUSTERING
} PROTOCOL

\begin{tabular}{|l|l|}
\hline Lakshmi N & Manjunath H R \\
Dept. of computer science & Asst.Professor,Dept. of Computer \\
and Engineering, & Science and Engineering, \\
Shridevi Institute of Engineering & Shridevi Institute of Engineering \\
and Technology, Tumkur, India & and Technology, \\
lakshmi15592@gmail.com. & Tumkur, India. \\
& manjunathdvg@gmail.com \\
\hline
\end{tabular}

Abstract-In wireless sensor network life time, the energy efficiency is the determinant aspect. To reduce the energy consumption and to improve the network lifetime, various routing protocols are used. In case of hierarchical clustering, the process of aggregation and fusion of data is performed, which is an efficient way to reduce the overall energy consumption within the cluster. Base Station Controlled Dynamic Clustering Protocol (BCDCP) is one of the example of hierarchical clustering protocols in which Cluster Heads(CH) perform Aggregation and Fusion of data.

This paper proposes a Fuzzy Logic technique of Artificial Intelligence to be applied to the conventional $B C D C P$ to reduce the energy consumption and also improves the efficiency which is the main objective and this leads to improve the lifetime of the network. Such an environment is created and the same is simulated. Energy Consumption as well as the Lifetime of the Conventional and Fuzzy-Based BCDCP are compared by plotting the graphs.

Keywords- Wireless Sensor Network, Routing protocols, Hierarchical clustering, BCDCP, Artificial Intelligence, Fuzzy logic.

\section{INTRODUCTION}

In conventional $\mathrm{BCDCP}$ only one parameter at $\mathrm{CH}$ selection[3] and also while attaching the nodes to the CH's is considered that are Energy level and distance respectively.Due to this reason conventional BCDCP is not much efficient. As the improvement the efficiency is one of the important thing to be taken care in WSN[1], the technique named Fuzzy Logic[6] [7] [8] of Artificial Intelligence[2] is applied to the conventional BCDCP in order to improve its lifetime. Siva D. Muruganathan [5]proposed a centralized routing protocol called Base Station Controlled Dynamic Clustering Protocol (BCDCP), which distributes the energy dissipation evenly among all sensor nodes to improve network lifetime and average energy savings. Fuzzy logic is a superset of conventional logic that has been extended to handle the concept of partial-truth values between the Boolean dichotomy of true and false. Fuzzy logic usually takes the form of a fuzzy reasoning system and its components are fuzzy variables, fuzzy rules and a fuzzy inference engine [11].Fuzzy Logic is designed to solve problems in the same way that humans do by considering all available information and making the best possible decision for the given input.

The advantage of applying Fuzzy technique is to consider more parameters when compare to the conventional approach in which only one parameter is considered at both the levels. Maximization of network lifetime is achieved with the proposed approach which is the objective of this paper.

\section{MOTIVATION}

The hierarchical clustering scheme uses spanning tree based approach to produce cluster with certain properties. However, energy efficiency is not addressed in this work[3]. One of the basic protocol of hierarchical clustering i.e., LEACH is considered and detailed study is taken place,the limitations are listed[4]. These limitations can be overcome by BCDCP which is the protocol that is considered in this paper. The "Performance of BCDCP is compared with thet of LEACH which says that,BCDCP is more efficient than LEACH[5]. Then the study on the technique of Artificially Intelligence to improve the performance of BCDCP is done which says that", Fuzzy-logic-based clustering approach with an extension to the energy 


\section{Asia Pacific Journals}

predication has been proposed to prolong the lifetime of WSNs by evenly distributing the workload. The simulation results show that the proposed approach is more efficient than other distributed algorithms could be further applied to large-scale wireless sensor networks" [12].So to achive the objective of this paper Fuzzy Logic of AI is applied to the conventional BCDCP.

\section{APPROACH}

The work proposes a novel technique i.e., Fuzzy Logic of AI to improve the efficiency of BCDCP. Jprowler simulator is used to simulate the result of both conventional as well as fuzzy-based BCDCP. Simulated results are then compared to check the amount of energy consumption and lifetime of the network. Considering more than one parameter in clustering a network is essential to maximize the lifetime of the network is clearly exhibited in this paper.

\section{A. Conventional BCDCP Approach}

Conventional BCDCP considers one parameter at $\mathrm{CH}$ selection and one parameter while attaching the nodes to the CHs. None other than energy is taken into consideration which which we can say as less efficient. The figure-1 below shows the conventional BCDCP. It forms the cluster by consuming number of nodes, number of $\mathrm{CH}$ 's and range of the network as inputs. Cluster is formed based on the consideration of energy at $\mathrm{CH}$ selection and distance at attaching nodes to $\mathrm{CH}$ 's.

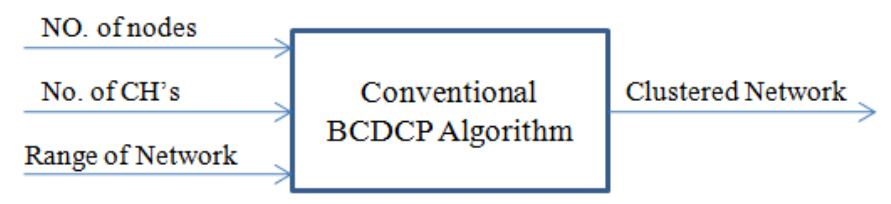

Figure-1: conventional BCDCP

The cluster formation in conventional BCDCP for 80 number of nodes, $5 \mathrm{CH}$ 's and for network range 100 is shown in the below figure- 2 .

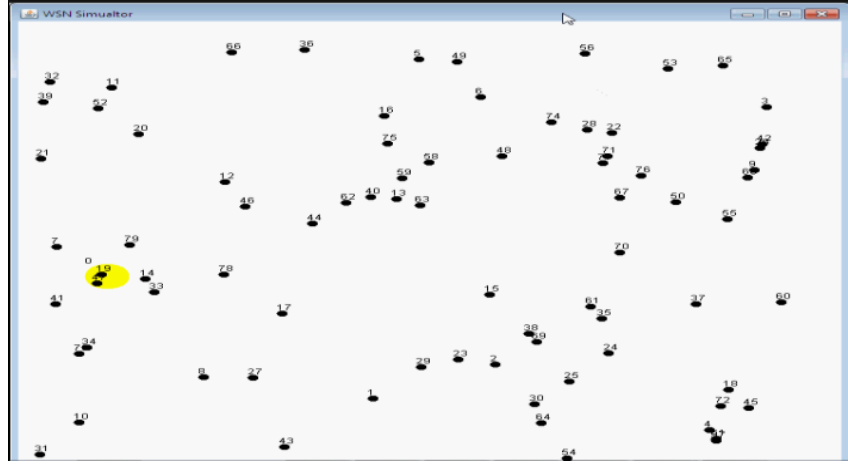

Figure-2: Cluster formation in conventional $B C D C P$

\section{B. Fuzzy Applied BCDCP Approach}

A centralized routing protocol called Base Station Controlled Dynamic Clustering Protocol (BCDCP), which distributes the energy dissipation evenly among all sensor nodes to improve network lifetime and average energy savings.

The main goal here is, to improve the efficiency of the conventional BCDCP algorithm which in turn maximizes the network lifetime by using the technique called as fuzzy logic of AI.

Fuzzy logic[9] [10] is applied to the conventional BCDCP algorithm at two levels:

- $\mathrm{CH}$ selection

- Attaching the nodes to CH's

The parameters considered in the $\mathrm{CH}$ selection are:

- Transmission Time Rate(TTR)

- Remaining Battery Power of Sensors(RPS)

- $\quad$ Sleep Time Rate(STR)

The below figure- 3 shows the pictorial representation of the same:

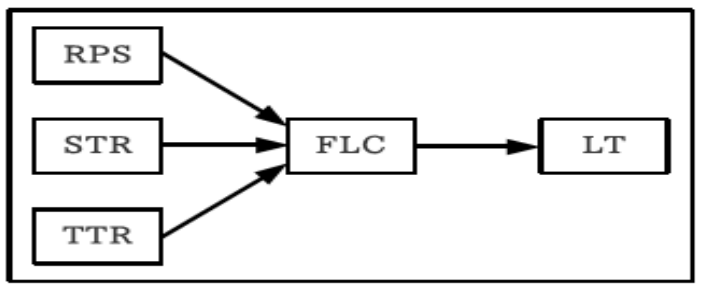

Figure-3:FLC at CH selection 
Asia Pacific Journals

The parameters considered in attaching the nodes to the respective CH's are:

- Distance

- Load

- Preference

The below figure- 4 shows the pictorial representation of the same:

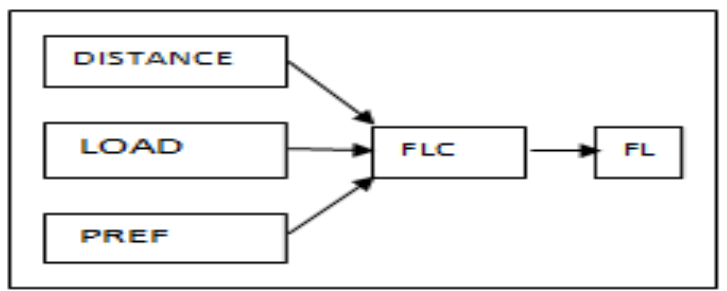

Figure-4:.FCL at attaching the nodes to $\mathrm{CH}$ 's

Diagrammatic representation of FCL application in the conventional BCDCP algorithm is shown in Figure-3 and Figure-4. Fuzzy logic is a superset of conventional logic that has been extended to handle the concept of partial-truth values between the Boolean dichotomy of true and false. Fuzzy logic[13] usually takes the form of a fuzzy reasoning. All the parameters are divided into three levels: for example, TTR is divided into few, medium and many. The same type of division is made to all other parameters too. The outcome to represent the node cluster-head election chance is divided into seven levels: very small, small, rather small, medium, rather large, large, and very large.

The fuzzy rule base currently includes rules like the following: " if the Transmission Time Rate(TTR) is high and the Remaining Battery Power of Sensors(RPS) is low and Sleep Time Rate(STR) is long then the node's cluster-head election chance is very large". Thus we use $33=27$ rules for the fuzzy rule base.

Here, we generate set of rules called as fuzzy rules, clustering is done by comparing the inputs with these set of rules. Finally, the output results are compared with that of the results tabulated without applying artificial intelligence. Increase in the efficiency of the conventional BCDCP by applying fuzzy logic technique of $\mathrm{AI}$ is then represented clearly with the help of graph.
The figure- 5 below shows the application of Fuzzy Logic at two levels of clustering and produces an efficient clustered network as output.

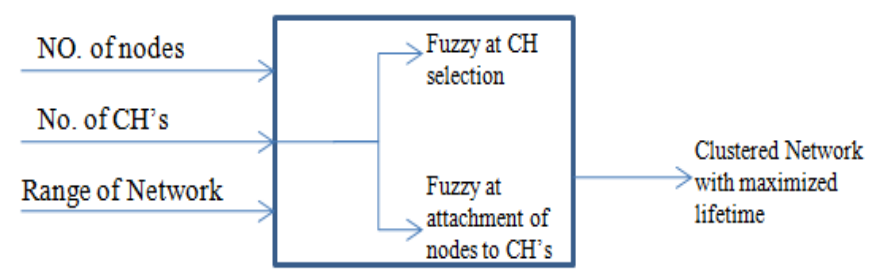

Figure-5: Fuzzy applied BCDCP

The cluster formation for 80 number of nodes, 5 $\mathrm{CH}$ 's and for network range 100 is shown in the below figure- 6 by applying the Fuzzy-technique of AI.

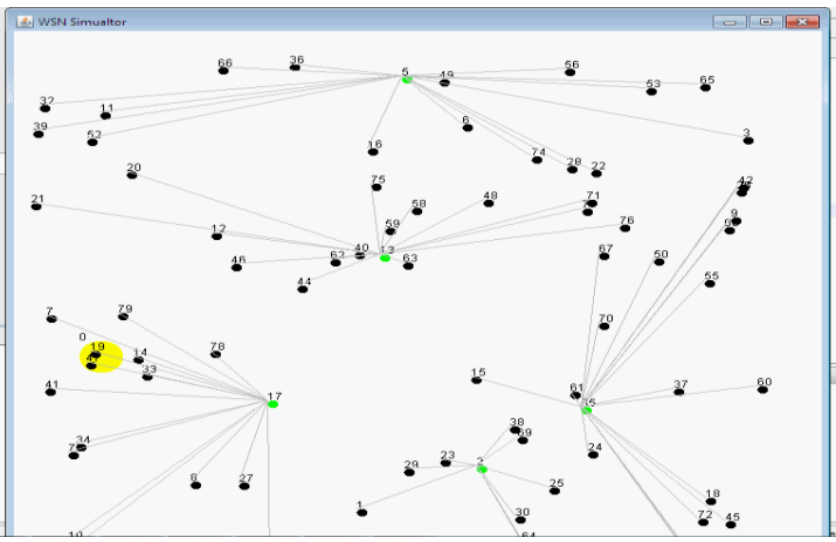

Figure-6:Cluster formation in Fuzzy Based BCDCP

\section{RESULTS AND DISCUSSION}

The simulator used here is Jprowler which is based on MATLAB and operates in probabilistic or deterministic mode. It also Provides better visualization capabilities. Below Graph-1 shows the graph of nodes versus energy consumption. It is very easy to say from the graph that, Fuzzy-Based BCDCP consumes less energy when compared to conventional BCDCP. 
ELK

Asia Pacific Journals

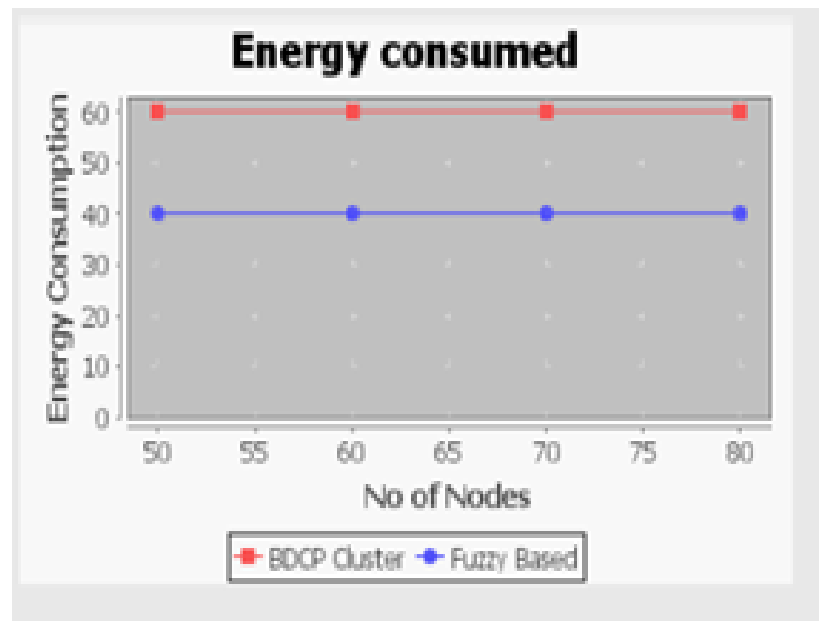

Graph 1:Graph of nodes versus energy consumption

Below Graph-2 shows the graph of nodes versus simulation time. It is very easy to say from the graph that, Fuzzy-Based BCDCP has maximum lifetime compared to conventional BCDCP.

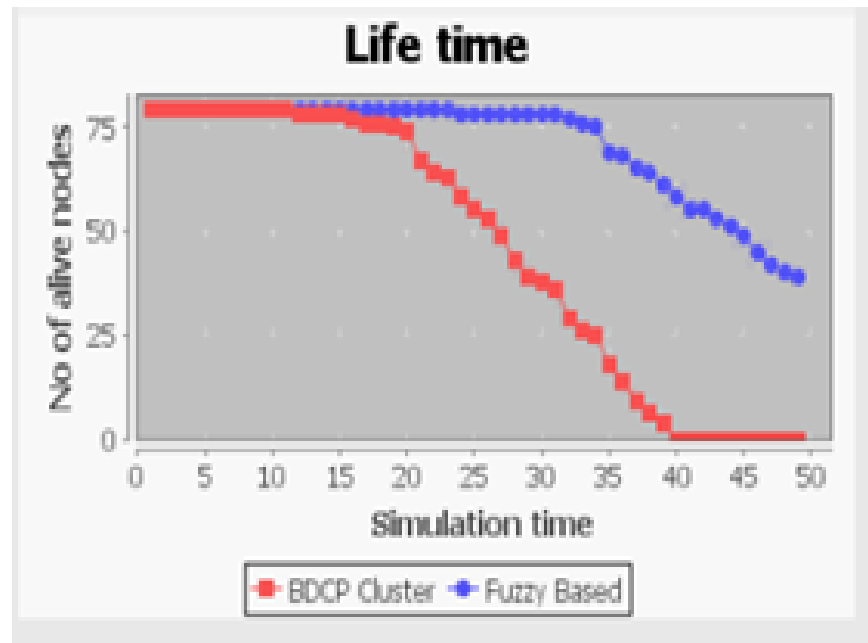

Graph -2: Graph of nodes versus simulation time

\section{CONCLUSION}

This paper proposes an efficient technique for improving the lifetime of conventional BCDCP by reducing the amount of energy consumption during network clustering. The simulation results show that the proposed approach is more efficient and could
ELK Asia Pacific Journals - Special Issue ISBN: 978-81-930411-5-4

be further applied to large-scale wireless sensor networks.

\section{ACKNOWLEDGMENT}

The authors would like to thank to Shridevi Institute Engineering and Technology, Tumkur for supporting this work.

\section{REFERENCE}

[1] K. Padmanabhan and P. Kamalakkannan "Energy Enhanced Base Station Controlled Dynamic Clustering Protocol for Wireless Sensor Networks". Journal of Advances in Computer Networks, Vol. 1, No. 1, March 2013.

[2] R.J.SOLOMONOFF,Member,IEEE "Some recent works in Artificial Intelligence," Proceedings of the IEEE,VOL. 54,NO. 12,December 1966.

[3] Vinay Kumar, Sanjay B. Dhok, Rajeev Tripathi and Sudarshan Tiwari, "A Review Study of Hierarchical Clustering Algorithms for Wireless Sensor Networks", IJCSI International Journal of Computer Science Issues, Vol. 11, Issue 3, No 1, May 2014.

[4] M. Madheswaran and R. N. Shanmugasundaram, "Enhancements of LEACH Algorithm for Wireless Networks: A Review" ICTACT Journal on Communication Technology, December 2013, volume: 04, Issue: 04.

[5] S.D.Muruganathan, D.C.F. Ma, R.I. Bhasin and A.O. Fapojuwoy, " A Centralized Energy Efficient Routing Protocol for Wireless Sensor Network ",in Proceedings of IEEE Radio Communication, March, 2005.

[6] Jong-Myoung Kim, Seon-Ho Park, Young-Ju Han and Tai-Myoung Chung, "CHEF: Cluster Head Election mechanism using Fuzzy logic in Wireless Sensor Networks," ISBN 978-895519-136-3,Feb. 17-20, 2008 ICACT 2008.

[7] Junpei Anno,Leonard Barolli, "A Cluster Head Selection method for Wirelwss Sensor Networks based on fuxxy logic," IEEE 2007.

[8] Indranil Gupta, Denis Riordan, Srinivas Sampalli "Cluster-head Election using Fuzzy Logic for Wireless Sensor Networks," 
Asia Pacific Journals

Proceedings of the 3rd Annual Communication Networks and Services Research Conference (CNSR'05) 0-7695-2333-1/05 2005 IEEE.

[9] Jin-Shyan Lee, Senior Member, IEEE, and WeiLiang Cheng, "Fuzzy-Logic-Based Clustering Approach for Wireless Sensor Networks Using Energy Predication," IEEE SENSORS JOURNAL, VOL. 12, NO. 9, SEPTEMBER 2012.

[10] Haifeng Jiang, Yanjing Sun, Renke Sun, and Hongli $\mathrm{Xu}$, "Fuzzy-Logic-Based Energy Optimized Routing for Wireless Sensor Networks," International Journal of Distributed Sensor Networks.

[11] Michele Pirovano1,2 1Department of Computer Science, University of Milano, Milano, Italy 2Dipartimento di Ingegneria
Elettronica e Informazione, Politecnico di Milano, Italy, "The use of Fuzzy Logic for Artificial Intelligence in Games" December 7, 2012. [1] Vinay Kumar, Sanjay B. Dhok Rajeev Tripathi and Sudarshan Tiwari,

[12] Jin-Shyan Lee, Senior Member, IEEE, and Wei-Liang Cheng, "Fuzzy-LogicBased Clustering Approach for Wireless Sensor Networks Using Energy Predication" IEEE Sensors Journal, Vol. 12, No. 9, September 2012.

[13] Ge Rana,*, Huazhong Zhangb, Shulan Gongc, "Improving on LEACH Protocol of Wireless Sensor Networks Using Fuzzy Logic", Journal of Information \& Computational Science 7: 3 (2010) 767-775 Available at http://www.joics.com 